\title{
Feststellungen und Forderungen eines nebenamtlich tätigen Schularztes
}

\author{
H. Häfliger
}

Leitender Schularzt der Stadtschulen Luzern, Würzenbachstrasse 17, 6006 Luzern

\begin{abstract}
In der Stadt Luzern haben vor drei Jahren sechs frei praktızierende Kinderärzte den schulärztlichen Dienst ïbernommen, der vorher während Jahrzehnten vom hauptamtlichen Stadtarzt versehen worden war. Die Schulhäuser wurden den einzelnen schulärzten zugeteilt, die Untersuchungen finden in der Arztpraxis statt. Ein Grossteil der Schüler sind dem im gleichen Quartier tätigen Kinder- und Schularzt bereits bekannt, was die Untersuchungen erleichtert und Doppelspurigkeiten vermeiden hilft. Auch die Kosten konnten so ohne Leistungsabbau erheblich gesenkt werden. Die bisherigen Erfahrungen sind auch aus der Sicht der Schüler, Eltern und Lehrer eindeutig positiv.
\end{abstract}

Als lettender Schularzt der stadt Luzern befasse lch mich seit der Uebernahme dieses Nebenamtes selbstverständlich mehir und ,regelmässig mit Fragen des schulärztlichen Dienstes und allgemein der Sozial- und Präventivmedizin. Dies erlaubt mir, folgende vier Feststellungen zu machen:

1) Von mehr als 1800 Schulärzten der Schweiz sind uber 98 prozent nebenamtlich tatig, in ihrer grossen Mehrhelt Allgemeinpraktiker und in der Regel ohne spezielle Ausbildung in Präventivmedizin.

2) Viele Kollegen äussern ein gewisses Unbehagen iber den sinn der bis jetzt üblichen Art der schulärztlichen Relhenuntersuchungen. Darunter leidet oft auch die sualität.

3) Auch in zukunft müssen die schulärztlichen Aufgaben in den meisten nrten der Schweiz von frei praktizierenden Aerzten im Nebenamt übernommen werden und zwar aus gesundheitspolitischen und schulpolitischen Grinden, vor allem auch aus $f i-$ nanzpolitischen Ueberlegungen und nicht zuletzt aus standespolitischer sicht.

4) Für neue oder andere Aufgaben, die von einem modernen schulärztlichen Dienst übernommen werden sollen, sind die schulärzte, von Ausnahmen abgesehen, nicht vorbereitet und nicht ausgebildet, also nicht kompetent oder besser noch nicht kompetent genug.

Aus den vier Feststellungen folgere ich drel Forderungen, welche eine sinnvolle, den heutigen Bedürfnissen angepasste, jedoch nicht überstürzte Reform des schulärztlichen Dienstes in der schweiz in seiner gegenwärtigen Vielfalt ermöglichen könnten:

1) Erarbeitung eines realistischen Konzeptes für den etappenweisen Aufbau elnes modernen schulärztlichen Dienstes möglichst in der ganzen Schweiz, in welchem regional oder föderalistisch bedingte Unter- schiede oder Schwerpunkte Platz haben.

2) Die Initiative $\mathrm{zu}$ dieser Erneuerung muss von uns Schulärzten ausgehen. Die nebenamtlich tätigen Schulärzte müssen hierbei ernsthaft mitreden und mitbestimmen. Dies nicht nur wegen ihrer zahlenmässigen "vebermacht", sondern vor allem wegen ihrer oft jahrelangen Erfahrung und ihrem Sinn für das Mögliche und Machbare, damit die Reform nicht nur auf dem Papier stattfindet.

3) Neue Schulärzte müssen in ihre Aufgabe eingefüht werden und regelmässig sind Fortbildungsgelegenheiten zu schaffen, um für neue Aufgaben kompetent zu sein und Erfahrungen auszutauschen. Dies sind wir uns ja gewohnt von unserer hauptberuflichen Tätigkeit her.

Zusammenfassung:

Der schulärztliche Dienst in der Schweiz wird zur Hauptsache von nebenamtlich tätigen Aerzten in freier Praxis gelelstet, die meisten ohne spezielle Ausbildung. Reformbemühungen müssen dahingehen, diese Aerzte für ihre Aufgaben in einem modernen schulärztlichen Dienst kompetenter $\mathrm{zu}$ machen.

\section{Résume:}

Constations et exigences d'un médecin scolaire a titre de fonction secondaire

Le service medical scolaire en Suisse est assuré dans la majeure partie des cas a titre de fonction secondaire par des médecins travaillant dans leur cabinet privé; la plupart d'entre eux n'ont aucune formation spéciale. Les tendances réformatrices devraient amener ces médecins à une competence plus grande, afin de mieux assumer leur tâche dans un service médical scolaire moderne.

\section{Summary:}

Findings and suggestions made by a part-time school doctor

Medical care in Swiss schools is largely carried out by general practitioners, who do this work in addition to their regular duties; the majority of these doctors have received no specialized training for this work. Any attempts to reform the status quo must be directed towards enabling these doctors to do their work more efficiently in a modern medical service for schools. 